\title{
The Effect of Emotional Conflict on Attention Allocation: An Event-Related Potential Study
}

\author{
Mengyin Zhu',2, Jing Ma1,2, Qi Jiang1,2, Wenbo Luo ${ }^{3}$, Min Hou, ${ }^{1,2}$, Xiao Chen ${ }^{1,2}$ \\ ${ }^{1}$ Institution of Mental Health Education, Southwest University, Chongqing, China \\ ${ }^{2}$ Faculty of Psychology, Southwest University, Chongqing, China \\ ${ }^{3}$ Laboratory of Cognition and Mental Health, Chongqing University of Arts and Sciences, Chongqing, China \\ Email: jiangqi@swu.edu.cn, wenbo9390@sina.com
}

Received 21 December 2014; accepted 30 January 2015; published 6 February 2015

Copyright (c) 2015 by authors and Scientific Research Publishing Inc.

This work is licensed under the Creative Commons Attribution International License (CC BY). http://creativecommons.org/licenses/by/4.0/

(c) (i) Open Access

\section{Abstract}

Attention allocation research frequently focuses on the valence of emotion. However, there is increasing evidence to indicate that arousal may be more relevant at some stages of affective processing. The present study employed a combined priming and cue-target paradigm and examined event-related potentials (ERPs) in order to explore the effects of emotional conflict of arousal on attention allocation. The background context of arousal was manipulated (using photos of facial expressions) while subjects performed a cognitive task in which a central arrow cue indicated the location of a peripheric target square, and a response was made according to the direction of the square. There was no main effect between incongruent emotion and congruent emotion in the response time, which means the resolution of the emotional conflict facilitate the participant behavioral response. There was a main effect of different emotional states on ERP component. In the present study, incongruent arousal also triggered greater positive potential compared with congruent arousal.

\section{Keywords}

Arousal, Congruency, Incongruency, Attention Allocation, Event-Related Potentials, Emotional Conflict

\section{Introduction}

Affective facial pictures, which vary in terms of valence and arousal, have been utilized as a background mani- 
pulation in some studies. These studies indicate that cognitive processes are influenced by such background information [1] [2]. Most researchers have focused their attention on valence. However, there is evidence to suggest that arousal may be more relevant at some stages of affective processing [3]-[5]. The present study adopted an arousal priming paradigm wherein an affective priming face was followed by another affective face (target face) after a certain interval. This paradigm elicits a congruent or incongruent arousal state, reflected by differences in reaction time (RT) and event-related potentials associated with the different arousal states [6]. Both the priming and target facial picture provide an emotional background.

Since the cognitive conflict and the mechanism of conflict control have been explored broadly, the researchers have extended their attention to the affective conflict [7] by employing various paradigms, such as the emotion AX-Continuous Performance [8], Word-Stroop task [9]-[11], the emotional Stroop task [12] and the emotional Flanker task [13]. These researches indicated that the reaction times (RTs) on emotional incongruent trials are significantly longer than those on emotional congruent trials [7] [8] [13]. Interestingly, some studies have revealed that RTs on emotional incongruent trials are longer when preceded by an emotional congruent trial than when preceded by an emotional incongruent trial, as the same, RTs on emotional congruent trials are shorter when preceded by an emotional congruent trials than those when preceded by an emotional incongruent trial [9] [14]. These findings show that there may be emotional conflict adaptation to reduce the conflict by adjusting their subsequent performance when the participants were involved in emotional conflict.

Overt orienting is another frequently used manipulation in orienting research [15]. This may involve guidance using a central cue. For instance, this cue, which may be embedded within a facial picture, indicates where the target may appear at the periphery of the picture. The response to the orienting task may be different depending on the congruence or incongruence of dynamically changing background arousal.

Event-related potentials (ERPs) are known for their high temporal resolution, and so this method is well suited to the study of attentional orientation in congruent and incongruent arousal states. The P1 component is often used as an index of the early processing of stimuli in the visual cortex, as it is sensitive to non-specific increases in spatial attention [16]. It has been suggested that P1 reflects the process of selective sensory gating in the visual pathways during spatial attention. The P1 amplitude is enhanced during increases in temporal attention after the prime stimuli, and is associated with temporal cues and increased targets [17]. Late positive potential (LPP), which occurs several hundred milliseconds after stimulus onset (about $300 \mathrm{~ms}$ - $600 \mathrm{~ms}$ ), is another relevant component, distributed in the centro-parietal scalp region. It reflects an automatic increase in attention to visual emotional stimuli, and is mediated by the valence and arousal of affective pictures [18]. The emotional intensity of a stimulus will modulate the amplitude of the LPP. Emotional stimuli with positive or negative valence elicits a larger LPP than neutral stimuli [19], and more arousing neutral valence pictures trigger a larger LPP than those that are less arousing [20].

In a priming paradigm, the priming stimulus is presented first, followed by the target stimulus. When the priming stimulus is presented, then the individual will encode some of the attributes of the stimulus and activate the corresponding brain regions, which prepare the individual for the upcoming target stimulus. If the following target stimulus shares some common attributes with the previous priming stimulus, then the processing of the target stimulus will be enhanced [21]. However, the response will be inhibited if the attributes between the priming and target stimuli are different. Hence, priming may facilitate or inhibit subsequent processing of a target stimulus [22].

In the present study, participants were required to complete an experiment that comprised a combined priming and cue-target paradigm. Specifically, a cue arrow was embedded within a priming facial picture, which indicated the location of a square presented in the periphery of the subsequent target picture. The target picture was either congruent or incongruent with the priming picture in terms of arousal. As mentioned above, congruent arousal pictures may activate similar brain regions which facilitate the processing of the target picture, whereas incongruent pictures, with little or no overlap, may hinder processing of the target [23]. In our experiment, the paradigm is a little different with those paradigm mentioned before, we hypothesize that there will be the same effect occurred in our paradigm: the conflict condition which means the incongruent condition will employ more cognitive resource to resolve the emotional conflict, then the conflict condition will elicited more positive late ERP amplitudes than those elicited by non-conflict arousal photos. Since the conflict condition required more cognitive resource, then the participant may contribute more resource on the attention task, so the RTs is shorter in the incongruent condition than those in the congruent condition. 


\section{Material and Methods}

\subsection{Subjects}

Seventeen right-handed, healthy college students with normal or corrected-to-normal visual acuity were recruited to participate in the study ( 6 males, $\mathrm{M}=21.5$ years, $\mathrm{SD}=1.64 ; 11$ females, $\mathrm{M}=21$ years, $\mathrm{SD}=1.55$ ). All subjects gave written informed consent to participate and were paid for taking part. The study protocol was approved by the relevant ethics institution.

\subsection{Stimulus Materials}

Thirty gray scale pictures were selected from the native Chinese Facial Affective Picture System [24]. Ten of these were selected for the practice experiment to familiarize participants with the task procedure ( 5 priming photos [ 3 female, 2 male, arousal $5.06 \pm 0.147$, valence $4.41 \pm 0.51$ ], and 5 target photos [3 female, 2 male, arousal $5.00 \pm 0.23$, valence $5.00 \pm 0.23]$ ). The remaining 20 photos were used in the formal experiment (10 high arousal photos [ 5 male, arousal $8.00 \pm 0.41$; 5 female, arousal $7.27 \pm 0.28$ ], and 10 low arousal photos [5 male, arousal $4.00 \pm 0.41 ; 5$ female, arousal $3.33 \pm 0.24]$. There was a significant difference between the high and low arousal photos $(\mathrm{p}=0.001)$, with no difference in valence $(\mathrm{p}=0.187)$. The red (guide) arrow had a visual angle of $2^{\circ} \times 0.3^{\circ}$, and RGB values were $255,0,0$. Square 1 was light in color, with a visual angle of $1.2^{\circ} \times 1.2^{\circ}$, and RGB values of $185,225,225$. Square 2 was dark in color, with a visual angle of $1.2^{\circ} \times 1.2^{\circ}$, and RGB values of $0,225,225$. The prime and target facial photos were displayed in the center of the screen with a visual angle of $10^{\circ} \times 10^{\circ}$. E-prime 1.1 software (Psychology Software Tools, Inc., USA) was used to present the stimuli and record RT.

\subsection{Procedure}

The experiment was conducted in a sound-attenuated room. The subjects were seated approximately $80 \mathrm{~cm}$ from the computer screen. The procedure for one trial is depicted in Figure 1.

Each trial was initiated by a $300-500 \mathrm{~ms}$ presentation of a small white fixation on a black screen. Then, an emotional (low or high arousal) facial expression photo (priming stimulus) was presented in the center of the screen for $200 \mathrm{~ms}$. A red arrow embedded in the center of the photo indicated the location of the square that followed (square 2). In the priming photo, two squares (square 1) were located on the left and right side of the photo. The priming photo was replaced by a blank black screen presented for $100-150 \mathrm{~ms}$ [6], followed by the target photo (low or high arousal) for $1000 \mathrm{~ms}$. Two squares were presented at the same location as those in the priming photo, but the color of one square (square 2) was darker than square 1.

The study comprised four conditions: low arousal prime followed by low arousal target (L-L); low arousal prime followed by high arousal target (L-H); high arousal prime followed by low arousal target (H-L); and high arousal prime followed by high arousal target $(\mathrm{H}-\mathrm{H})$. The L-L and H-H conditions were congruent in arousal,

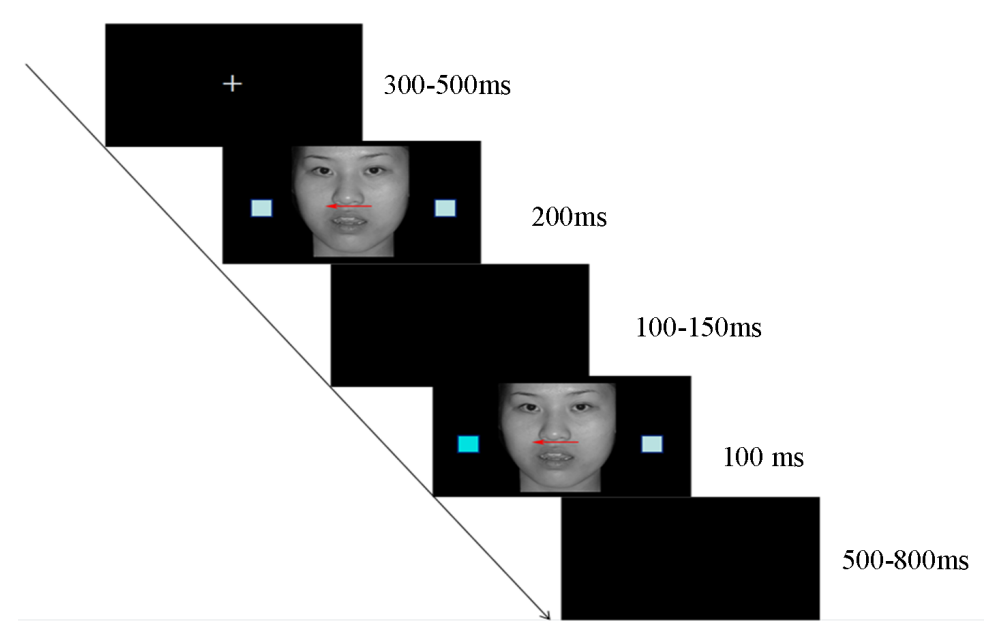

Figure 1. Example of experimental sequence for one trial. 
and the L-H and H-L conditions were incongruent.

Subjects responded to the location of square 2, which may have been in the position indicated by the direction of the arrow in the prime photo, or in the opposite direction. Half of the subjects pressed the "G" key if square 2 appeared on the left side of the photo, and " $\mathrm{H}$ " if it appeared on the right side. The responding keys were reversed for the other half of the participants. There was an $80 \%$ possibility that the red arrow indicated the correct position of square $2 \%$, and a $20 \%$ possibility that square 2 appeared on the opposite side to that indicated by the arrow. The inter-stimulus interval was $500-800 \mathrm{~ms}$.

The experiment consisted of five blocks. Each block had 80 trials, with $80 \%$ valid cue indication and $20 \%$ invalid cue indication in each block. Both the direction of the cue and the location of the target square were presented an equal number of times on the left and the right. There were 20 trials prior to the experiment in order to familiarize subjects with the experimental procedure.

\subsection{ERP Recording and Data Processing}

The electroencephalogram (EEG) was recorded from 58 scalp sites using an elastic cap (Brain Products, Munich, Germany). Two electrodes were placed lateral to the external canthi to record horizontal electrooculogram (HEOG). Another two electrodes were placed at the supra- and infra-orbital of the left eye to record vertical electrooculogram (VEOG). All electrodes were referenced to a left mastoid electrode and re-referenced offline to the average of the left and right mastoids. The EEG and EOG data were amplified using a DC- $100 \mathrm{~Hz}$ bandpass and continuously sampled at $500 \mathrm{~Hz} /$ channel. All inter-electrode impedances were maintained below $5 \mathrm{k} \Omega$. All data analyses were conducted using ERPLAB Toolbox (http://www.erpinfo.org/erplab) and EEGLAB Toolbox [25] (http://sccn.ucsd.edu/eeglab/), which are freely available, open source, Matlab-based packages for EEG/ERP data analysis. The EEG data were high-pass filtered at $0.1 \mathrm{~Hz}$ and low-pass filtered at $30 \mathrm{~Hz}$. Epochs containing blinks, eye movements, or other artifacts were excluded from further processing (amplitudes exceeding $\pm 100 \mathrm{uV}$ ).

The recorded ERPs, which were time-locked to the onset of the target photos, were assessed relative to a 150 ms pre-stimulus baseline (i.e., $150 \mathrm{~ms}$ prior to the presentation of the priming photo). The averaged epoch for ERPs was $1000 \mathrm{~ms}$, including the $150 \mathrm{~ms}$ baseline. In the present study, the mean amplitudes of the P1 and LPP components were analyzed. The following electrodes were used for analysis of the P1 component: O1, O2 and Oz. The electrode sites used for the LPP analysis were: $\mathrm{Cz}, \mathrm{CPz}, \mathrm{Pz}, \mathrm{POz}$ and Pzi which were averaged from CP1, CP2, CPz, P1, Pz, P2, PO1, POz, PO2 [Figure 2]. Due to the complexity of the LPP component, it was divided into 300 - $400 \mathrm{~ms}, 400$ - $500 \mathrm{~ms}, 500$ - $600 \mathrm{~ms}$ time windows.

The ANOVA for the ERPs evoked by the target stimuli during the time range of the P1 component included four factors: arousal emotion (congruent, incongruent), cue validity (valid, invalid), target location (left, right) and electrode location (O1, O2, Oz). The ANOVA for the ERPs evoked by the target stimuli during the time range of the LPP component included the same four factors with the exception that electrode locations were $\mathrm{Cz}$, $\mathrm{CPz}, \mathrm{Pz}, \mathrm{POz}$ and Pzi. The Greenhouse-Geisser method was used to correct the degrees of freedom of the F-ratio when violations of sphericity may have occurred. A Bonferroni correction was employed for multiple comparisons of behavioral and ERP data

\section{Results}

\subsection{Behavioral Data}

Trials with correct responses and RTs between $250 \mathrm{~ms}$ and $1000 \mathrm{~ms}$ were included in the RT analysis. Those data with RTs less than 250 ms were replaced with the mean RT for that subject. There was no main effect on incongruent and congruent emotional states $(F[1,16]=0.20, \mathrm{p}=0.660$, partial $\eta 2=0.012)$, which shows there was no difference between the different emotional states.The three-way repeated measures ANOVA results revealed a main effect of cue validity $(\mathrm{F}[1,16]=17.82, \mathrm{p}<0.01$, partial $\eta 2=0.527$, valid cue $\mathrm{M}=461.31$, $\mathrm{SD}=$ 11.96, invalid cue $\mathrm{M}=475.53$, SD = 13.07; Table 1 ). There was also a tendency for an interaction effect between arousal emotion, cue validity and target location $(F[1,16]=3.35, p=0.086$, partial $\eta 2=0.173)$. This indicates that the invalid cue decreased RT, different arousal states interacted with cue validity, and target location influenced RT. No other main effects or interaction effects were revealed. 

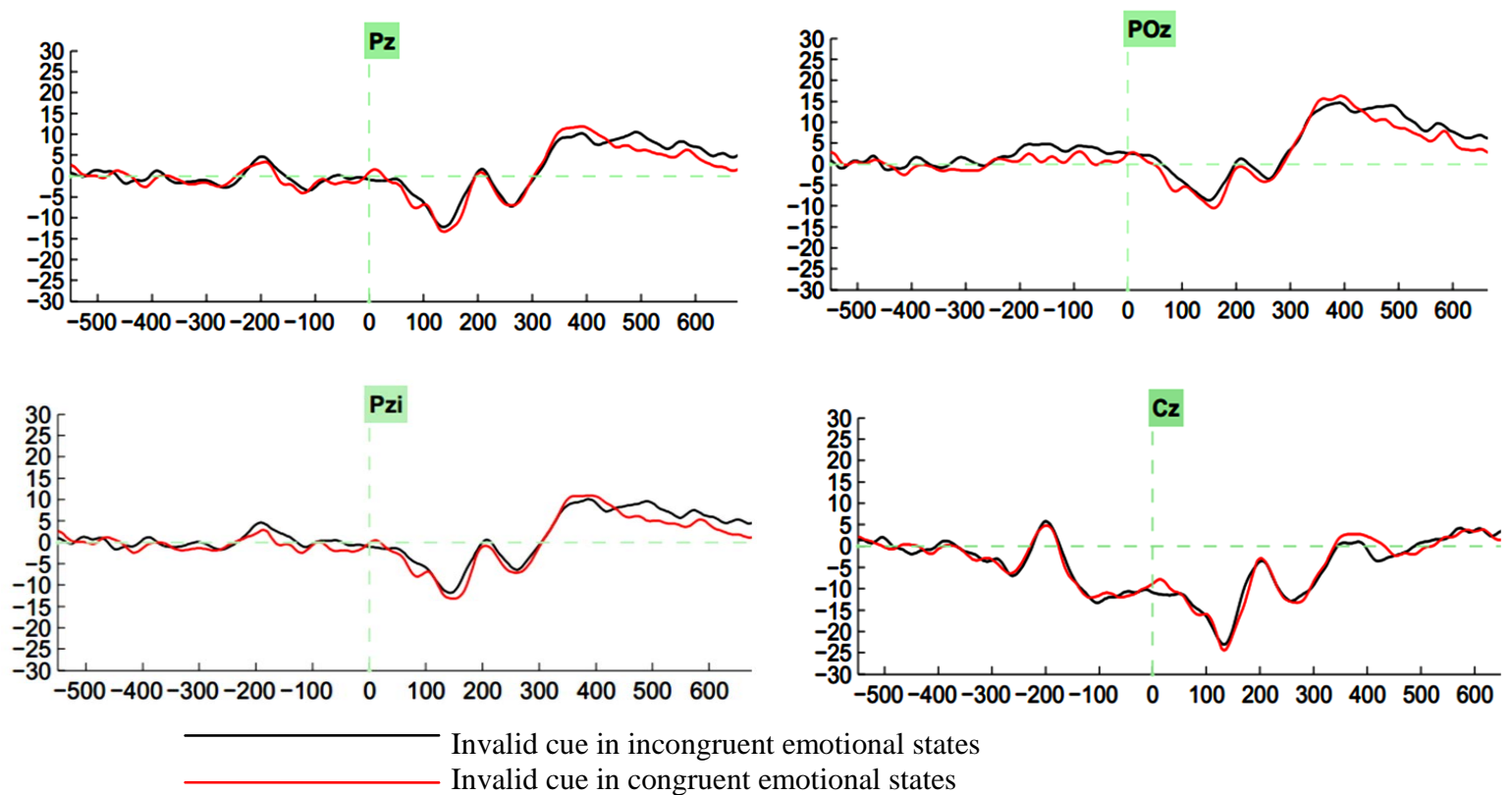

Figure 2. Grand average of target-elicited ERP waveforms at central-parietal sites.

Table 1. RT (ms) for each trial condition.

\begin{tabular}{ccccccc}
\hline & \multicolumn{3}{c}{ Main effect } & \multicolumn{3}{c}{ Interaction } \\
& V & I & V-L & V-R & I-L & I-R \\
\hline Mean & 461.31 & 475.54 & 463.68 & 458.94 & 474.74 & 476.33 \\
SD & 11.96 & 13.07 & 12.13 & 12.19 & 13.00 & 14.04 \\
\hline
\end{tabular}

a. V, valid cue; I, invalid cue; V-L, valid cue $\times$ direction left; V-R, valid cue $\times$ direction right; I-L, invalid cue $\times$ direction left; I-R, invalid cue $\times$ direction right.

\subsection{ERP Data}

\subsubsection{P1 Component}

The repeated measures ANOVA revealed no main effect on emotional arousal, cue validity, target location and electrodes and the other interaction on those factors, but there were significant interaction among emotional arousal, cue validity and target location $(\mathrm{F}[1,16]=4.65, \mathrm{p}<0.05$, partial $\eta 2=0.23)$, which provide electrophysiological evidence for the behavioral result, as it mentioned in the behavioral part that there were a tendency for an interaction effect between arousal emotion, cue validity and target location, the ERP data provide more concrete result. Specifically, in the target left and cue valid condition, the amplitude in the incongruent emotional arousal state $(-2.26 \pm 1.78 \mathrm{uV})$ was much bigger than that in the congruent state $(-1.58 \pm 2.26)$, while in the same target condition and emotional states, the cue invalid condition $(-2.76 \pm 1.80 \mathrm{uV})$ trigger much bigger amplitude than that in the cue valid state $(-1.50 \pm 2.80 \mathrm{uV})$, which indicate that the incongruent emotional state will trigger stronger amplitude than that in the congruent state, but the cue invalid condition will facilitate the amplitude of the component. There was also leaning towards significant interaction between emotional states and electrode sites $(\mathrm{F}[2,32]=2.19, \mathrm{p}=0.12$, partial $\eta 2=0.12)$.

\subsubsection{LPP Component}

$300-400$ ms. There were significant main effects of electrode sites $(F[4,64]=14.72, \mathrm{p}=<0.01$, partial $\eta 2=$ 0.819). In addition, the main effect of congruent and incongruent arousal $(\mathrm{F}=[1,16]=3.05, \mathrm{p}=0.1$, partial $\eta 2=$ 0.160 ) did not reach the traditional level of significant, but it resides on the edge of significance. There was also a trending towards significance for an interaction between emotional states and cue validity $(F[1,16]=3.15, p=$ 
0.09 , partial $\eta 2=0.165$ ).

400 - 500 ms. There were significant main effects of emotional states $(\mathrm{F}[1,16]=6.64, \mathrm{p}=<0.05$, partial $\eta 2=$ $0.293)$, and of electrode sites $(\mathrm{F}[4,64]=19.77, \mathrm{p}=<0.01$, partial $\eta 2=0.553)$. This indicates that the incongruent emotional states triggered a larger positive LLP than congruent states, which is similar to the findings for the $\mathrm{P} 1$ component, for the incongruent state $(\mathrm{M}=7.90, \mathrm{SD}=2.04)$, the congruent state $(\mathrm{M}=6.09, \mathrm{SD}=1.97)$, for more details in Table 2. In addition, there was a significant interaction between location of the target and electrode sites $(\mathrm{F}[4,64]=9.00, \mathrm{p}=<0.01$, partial $\eta 2=0.360)$. The interaction among emotional states, cue validity and target location was tantalizingly close to significance $(\mathrm{F}[1,16]=2.88, \mathrm{p}=<0.109$, partial $\eta 2=0.153$ ).

500 - $600 \mathrm{~ms}$. The results for LLP in the 500-600 ms time window were similar to those in the $400-500 \mathrm{~ms}$ time window. There was a significant main effect of emotional states, $(\mathrm{F}[1,16]=5.33, \mathrm{p}=<0.05$, partial $\eta 2=$ 0.250). As in the $400-500 \mathrm{~ms}$ time window, there was a significant interaction between target location and electrodes, $(\mathrm{F}[4,64]=10.15, \mathrm{p}<0.01$, partial $\eta 2=0.388)$.In addition, there was significant interaction between cue validity and electrode sites $(\mathrm{F}[4,64]=2.71, \mathrm{p}<0.05$, partial $\eta 2=0.145)$.Compared with the $400-500$ time window, the interaction among emotional states, cue validity and target location was significant $(F[1,16]=5.42$, $\mathrm{p}=<0.05$, partial $\eta 2=0.253$ ). The simple effect of emotional states within cue invalid within right side target location was significant $(F[1,16]=4.48, \mathrm{p}=<0.05)$, there were also significant simple effect of emotional states within valid cue within right side target location $(F[1,16]=7.41, \mathrm{p}<0.05)$, which indicate that no matter what the cue validity is the right target location has stronger LPP amplitude in the incongruent emotional state.

\section{Discussion}

Unlike previous research, which has focused on the dimension of valence, the present study involved dynamic changes in arousal state, which may be more representative of daily life. In the dynamic change of congruent and incongruent arousal states which triggered conflict state in the incongruent condition. However, the current experiment used a combined cue-target and priming paradigm to explore the implicit effect of congruent or incongruent arousal photos on attentional orienting. There was no difference on the behavioral result between the incongruent state and congruent state, but the result of ERP component, especially the LPP indicate that the different emotional state triggered different wave forms which suggest that the process of the two emotions were different. Meanwhile, the ERP waveforms associated with attentional orienting were different under congruent and incongruent arousal conditions, suggests that the different arousal states influenced attentional orienting.

As it mentioned above that there was no difference on the behavioral result between the two emotional states, which means there was no conflict effect in the incongruent condition, in other words, there was emotional conflict adaptation, which is consistent with the previous study that the participant will strategically adjust their emotional information processing to reduce the conflict [9]. RT is an index to indicate the resolution of the conflict, and there was no different under the incongruent and congruent states on the RT in our study, which suggest there was conflict resolution in the process of incongruent arousal face.

The P1 component associated with an invalid cue indication was significantly different at the occipital lobe (compared with the valid cue): the amplitude of P1 was larger for the invalid cue condition than the valid cue condition, which is consistent with a previous study [26]. This may be due to a tendency to enhance the visual response to targets at an attended location [27] and possibly suppress information that occurs at an unattended location; less cognitive resources are required to process an attended stimulus than an unattended stimulus, result ing in greater brain potential for the invalid cue indication target.

Table 2. Mean amplitude (uV) of the electrode sites on different emotional states.

\begin{tabular}{ccc}
\hline $\begin{array}{c}\text { Emotional states } \\
\text { Electrode sites }\end{array}$ & Incongruent emotional state & Congruent emotional state \\
\hline $\mathrm{CPz}$ & 7.02 & 5.41 \\
$\mathrm{Cz}$ & 2.24 & 0.52 \\
$\mathrm{POz}$ & 11.46 & 9.76 \\
$\mathrm{Pz}$ & 10.13 & 8.11 \\
$\mathrm{Pzi}$ & 8.65 & 6.65 \\
\hline
\end{tabular}


The other ERP component we focused on was the LPP, which occurs approximately $300 \mathrm{~ms}$ following stimulus onset at the central-parietal midline [18]. It is sensitive to a number of contextual and regulatory factors. Foti and Hajcak found that the amplitude of the LPP was modulated in neutral and negative descriptions that preceded unpleasant pictures. Specifically, the LPP was reduced for unpleasant pictures which were preceded by a neutral compared with a negative description [28]. In another recent matching and repetition priming study, LPP was revealed to be reduced in response to an unprimed target, and a primed target was associated with an LLP of a greater magnitude [29] [30]. In our study, we observed a similar modulated effect of LPP in an arousal context. Brain potentials were more positive in arousal incongruent states than in congruent states at the central-parietal midline region. This indicates that both arousal and valence have a modulating effect on LPP. One convincing explanation is that the incongruent condition was similar to an unprimed task, and there was no arousal-related priming effect. In the congruent condition, the priming photo may have activated brain religions that overlapped with those activated by the target photo for congruent stimuli, and this overlap effect resulted in a more positive potential. In contrast, the priming cue may have enhanced processing of the target in the congruent trials, so a stronger LPP was observed.

Another interesting result of the current study was that there was a significant main effect of emotional states, $(\mathrm{F}[1,16]=5.33, \mathrm{p}=<0.05$, partial $\eta 2=0.250)$ in $500-600 \mathrm{~ms}$ for the LPP, which shows the mechanism of processing the incongruent emotion were different with congruent emotion. There were interaction $(F[1,16]=$ $5.42, \mathrm{p}=<0.05$, partial $\eta 2=0.253$ ) among emotional states, cue validity and target location in the $500-600 \mathrm{~ms}$ time window for the LPP, further analysis suggest that no matter what the cue validity was the effect of emotional conflict was significant different in right side target location, which shown the right side dominant. A possible explanation is that the priming associated with the congruent emotional state was reflective of residual activation of the neural cluster for the preceding face context which overlapped with the subsequent congruent arousal face [31]. This may have facilitated the processing of the target face, enabling the participant to use more cognitive resources to deal with the incongruent task, thereby resulting in greater positive potential for the incongruent emotional condition. For the right side dominance, the result is consistent with the previous studies [32] [33], according to Welsh and Elliott's response activation model, the cues activate response codes for movement in that direction, so the right side dominance occurred, a saccadic eye movements got similar results [34].

Our study was mainly concerned with the difference in brain potentials associated with congruent or incongruent arousal states. Some recent studies have focused on various emotional conflict conditions, primarily including four kinds of conflict: low-low conflict, low-high conflict, high-high conflict, high-low conflict [35] [36]. These studies have addressed the emotional adjustment in dynamic conflict conditions, which has enabled investigation of more precise processing such as dynamic situations and the processing of abundant information. This has informed our ideas for further investigation in which we aim to design a more precise experiment and pinpoint the mechanism of the processing of dynamic arousal states.

\section{Conclusion}

In summary, this study extends on previous investigations by focusing on the effect of arousal in a priming study, utilizing a dynamically changing arousal condition which is more representative of real life. There is no main effect between incongruent emotion and congruent emotion in the response time, which means the resolution of the emotional conflict facilitate the participant behavioral response; there is main effect of different emotional states on ERP component in the present study. Incongruent arousal also triggers greater positive potential compared with congruent arousal.

\section{Acknowledgements}

This study was supported by the Program project of social and humanity, Ministry of Education of China (SKXM2009062), the 211 Key Subject Construction Program of South West University (NSKD11053), the National Natural Science Foundation of China (30900399, 31170984), the China Postdoctoral Science Foundation (20100470227, 201104069).

\section{References}

[1] Albert, J., Lopez-Martin, S. and Carretie, L. (2010) Emotional Context Modulates Response Inhibition: Neural and Behavioral Data. Neuroimage, 49, 914-921. http://dx.doi.org/10.1016/j.neuroimage.2009.08.045 
[2] Muller, M.M., Andersen, S.K. and Keil, A. (2008) Time Course of Competition for Visual Processing Resources between Emotional Pictures and Foreground Task. Cerebral Cortex, 18, 1892-1899. http://dx.doi.org/10.1093/cercor/bhm215

[3] Carretie, L., Martin-Loeches, M., Hinojosa, J.A. and Mercado, F. (2001) Emotion and Attention Interaction Studied through Event-Related Potentials. Journal of Cognitive Neuroscience, 13, 1109-1128. http://dx.doi.org/10.1162/089892901753294400

[4] Dillon, D.G., Cooper, J.J., Grent-'t-Jong, T., Woldorff, M.G. and LaBar, K.S. (2006) Dissociation of Event-Related Potentials Indexing Arousal and Semantic Cohesion during Emotional Word Encoding. Brain Cognition, 62, 43-57. http://dx.doi.org/10.1016/j.bandc.2006.03.008

[5] Rozenkrants, B., Olofsson, J.K. and Polich, J. (2008) Affective Visual Event-Related Potentials: Arousal, Valence, and Repetition Effects for Normal and Distorted Pictures. International Journal of Psychophysiology, 67, 114-123.

[6] Hinojosa, J.A., Carretie, L., Mendez-Bertolo, C., Miguez, A. and Pozo, M.A. (2009) Arousal Contributions to Affective Priming: Electrophysiological Correlates. Emotion, 9, 164-171. http://dx.doi.org/10.1037/a0014680

[7] Chiew, K.S. and Braver, T.S. (2010) Exploring Emotional and Cognitive Conflict Using Speeded Voluntary Facial Expressions. Emotion, 10, 842-854. http://dx.doi.org/10.1037/a0019704

[8] Chiew, K.S. and Braver, T.S. (2011) Neural Circuitry of Emotional and Cognitive Conflict Revealed through Facial Expressions. Plos One, 6, 21-25. http://dx.doi.org/10.1371/journal.pone.0017635

[9] Egner, T., Etkin, A., Gale, S. and Hirsch, J. (2008) Dissociable Neural Systems Resolve Conflict from Emotional versus Nonemotional Distracters. Cerebral Cortex, 18, 1475-1484. http://dx.doi.org/10.1093/cercor/bhm179

[10] Haas, B.W., Omura, K., Constable, R.T. and Canli, T. (2006) Interference Produced by Emotional Conflict Associated with Anterior Cingulate Activation. Cognitive, Affective, \& Behavioral Neuroscience, 6, 152-156. http://dx.doi.org/10.3758/CABN.6.2.152

[11] Haas, B.W., Omura, K., Constable, R.T. and Canli, T. (2007) Emotional Conflict and Neuroticism: Personality-Dependent Activation in the Amygdala and Subgenual Anterior Cingulate. Behavioral Neuroscience, 121, 249-256. http://dx.doi.org/10.1037/0735-7044.121.2.249

[12] Kunde, W. and Mauer, N. (2008) Sequential Modulations of Valence Processing in the Emotional Stroop Task. Experimental Psychology, 55, 151-156. http://dx.doi.org/10.1027/1618-3169.55.3.151

[13] Fenske, M.J. and Eastwood, J.D. (2003) Modulation of Focused Attention by Faces Expressing Emotion: Evidence from Flanker Tasks. Emotion, 3, 327-343. http://dx.doi.org/10.1037/1528-3542.3.4.327

[14] Etkin, A., Prater, K.E., Hoeft, F., Menon, V. and Schatzberg, A.F. (2010) Failure of Anterior Cingulate Activation and Connectivity with the Amygdala during Implicit Regulation of Emotional Processing in Generalized Anxiety Disorder. American Journal of Psychiatry, 167, 545-554. http://dx.doi.org/10.1176/appi.ajp.2009.09070931

[15] Posner, M.I. (1980) Orienting of Attention. Quarterly Journal of Experimental Psychology, 32, 3-25. http://dx.doi.org/10.1080/00335558008248231

[16] Brown, S., van Steenbergen, H., Band, G.P.H., de Rover, M. and Nieuwenhuis, S. (2012) Functional Significance of the Emotion-Related Late Positive Potential. Frontiers in Human Neuroscience, 6, 128-134. http://dx.doi.org/10.3389/fnhum.2012.00033

[17] Correa, A., Lupianez, J., Madrid, E. and Tudela, P. (2006) Temporal Attention Enhances Early Visual Processing: A Review and New Evidence from Event-Related Potentials. Brain Research, 1076, 116-128. http://dx.doi.org/10.1016/j.brainres.2005.11.074

[18] Hajcak, G., Dunning, J.P. and Foti, D. (2009) Motivated and Controlled Attention to Emotion: Time-Course of the Late Positive Potential. Clinical Neurophysiology, 120, 505-510. http://dx.doi.org/10.1016/j.clinph.2008.11.028

[19] Hajcak, G., MacNamara, A. and Olvet, D.M. (2010) Event-Related Potentials, Emotion, and Emotion Regulation: An Integrative Review. Developmental Neuropsychology, 35, 129-155. http://dx.doi.org/10.1080/87565640903526504

[20] Weinberg, A. and Hajcak, G. (2011) The Late Positive Potential Predicts Subsequent Interference with Target Processing. Journal of Cognitive Neuroscience, 23, 2994-3007. http://dx.doi.org/10.1162/jocn.2011.21630

[21] Eder, A.B., Leuthold, H., Rothermund, K. and Schweinberger, S.R. (2012) Automatic Response Activation in Sequential Affective Priming: An ERP Study. Social Cognitive and Affective Neuroscience, 7, 436-445. http://dx.doi.org/10.1093/scan/nsr033

[22] Zhang, Q., Lawson, A., Guo, C. and Jiang, Y. (2006) Electrophysiological Correlates of Visual Affective Priming. Brain Research Bulletin, 71, 316-323. http://dx.doi.org/10.1016/j.brainresbull.2006.09.023

[23] Eddy, M.D. and Holcomb, P.J. (2009) Electrophysiological Evidence for Size Invariance in Masked Picture Repetition Priming. Brain Cognition, 71, 397-409. http://dx.doi.org/10.1016/j.bandc.2009.05.006

[24] Gong, X., Huang, Y.X., Wang, Y. and Luo, Y.J. (2011) Revision of the Chinese Facial Affective Picture System. Chi- 
nese Mental Health Journal, 25, 40-46.

[25] Delorme, A. and Makeig, S. (2004) EEGLAB: An Open Source Toolbox for Analysis of Single-Trial EEG Dynamics including Independent Component Analysis. Journal of Neuroscience Methods, 134, 9-21. http://dx.doi.org/10.1016/j.jneumeth.2003.10.009

[26] Luck, S.J., Hillyard, S.A., Mouloua, M., Woldorff, M.G., Clark, V.P. and Hawkins, H.L. (1994) Effects of Spatial Cuing on Luminance Detectability: Psychophysical and Electrophysiological Evidence for Early Selection. Journal of Experimental Psychology: Human Perception and Performance, 20, 887-904. http://dx.doi.org/10.1037/0096-1523.20.4.887

[27] Albares, M., Criaud, M., Wardak, C., Song, C.T.N., Ben Hamed, S. and Boulinguez, P. (2011) Attention to Baseline: Does Orienting Visuospatial Attention Really Facilitate Target Detection? Journal of Neurophysiology, 106, 809-816. http://dx.doi.org/10.1152/jn.00206.2011

[28] Foti, D. and Hajcak, G. (2008) Deconstructing Reappraisal: Descriptions Preceding Arousing Pictures Modulate the Subsequent Neural Response. Journal of Cognitive Neuroscience, 20, 977-988. http://dx.doi.org/10.1162/jocn.2008.20066

[29] Bobes, M.A., Martin, M., Olivares, E. and Valdes-Sosa, M. (2000) Different Scalp Topography of Brain Potentials Related to Expression and Identity Matching of Faces. Cognitive Brain Research, 9, 249-260. http://dx.doi.org/10.1016/S0926-6410(00)00003-3

[30] Schweinberger, S.R., Pfutze, E.M. and Sommer, W. (1995) Repetition Priming and Associative Priming of Face Recognition-Evidence from Event-Related Potentials. Journal of Experimental Psychology: Learning, Memory, and Cognition, 21, 722-736. http://dx.doi.org/10.1037/0278-7393.21.3.722

[31] Gentile, F. and Jansma, B.M. (2010) Neural Competition through Visual Similarity in Face Selection. Brain Research, 1351, 172-184. http://dx.doi.org/10.1016/j.brainres.2010.06.050

[32] Elliott, T.N.W.D. (2004) Effects of Response Priming and Inhibition on Movement Planning and Execution. Journal of Motor Behavior, 36, 200-211. http://dx.doi.org/10.3200/JMBR.36.2.200-211

[33] Welsh, T.N. and Elliott, D. (2004) Movement Trajectories in the Presence of a Distracting Stimulus: Evidence for a Response Activation Model of Selective Reaching. The Quarterly Journal of Experimental Psychology Section A, 57, 1031-1057. http://dx.doi.org/10.1080/02724980343000666

[34] Sheliga, B., Craighero, L., Riggio, L. and Rizzolatti, G. (1997) Effects of Spatial Attention on Directional Manual and Ocular Responses. Experimental Brain Research, 114, 339-351. http://dx.doi.org/10.1007/PL00005642

[35] Etkin, A., Egner, T., Peraza, D.M., Kandel, E.R. and Hirsch, J. (2006) Resolving Emotional Conflict: A Role for the Rostral Anterior Cingulate Cortex in Modulating Activity in the Amygdala. Neuron, 51, 871-882. http://dx.doi.org/10.1016/j.neuron.2006.07.029

[36] Mansouri, F.A., Tanaka, K. and Buckley, M.J. (2009) Conflict-Induced Behavioural Adjustment: A Clue to the Executive Functions of the Prefrontal Cortex. Nature Reviews Neuroscience, 10, 141-152. http://dx.doi.org/10.1038/nrn2538 
Scientific Research Publishing (SCIRP) is one of the largest Open Access journal publishers. It is currently publishing more than 200 open access, online, peer-reviewed journals covering a wide range of academic disciplines. SCIRP serves the worldwide academic communities and contributes to the progress and application of science with its publication.

Other selected journals from SCIRP are listed as below. Submit your manuscript to us via either submit@scirp.org or Online Submission Portal.
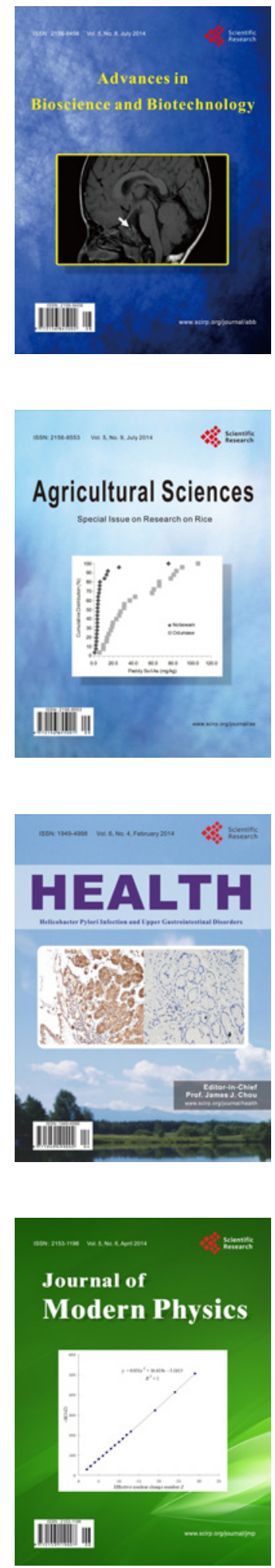
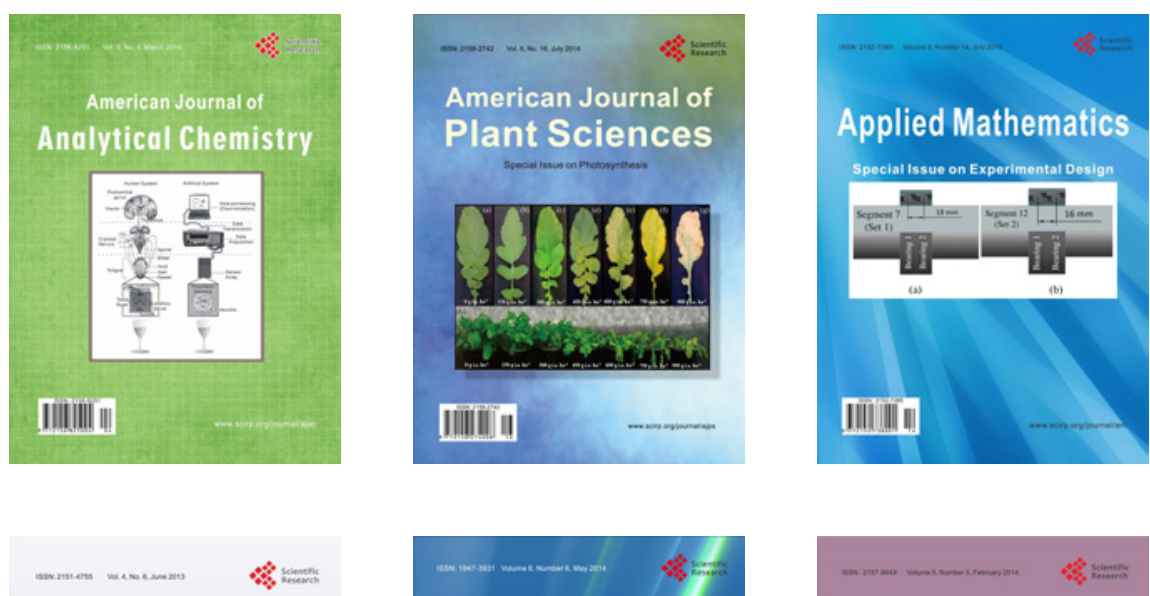

Creative Education
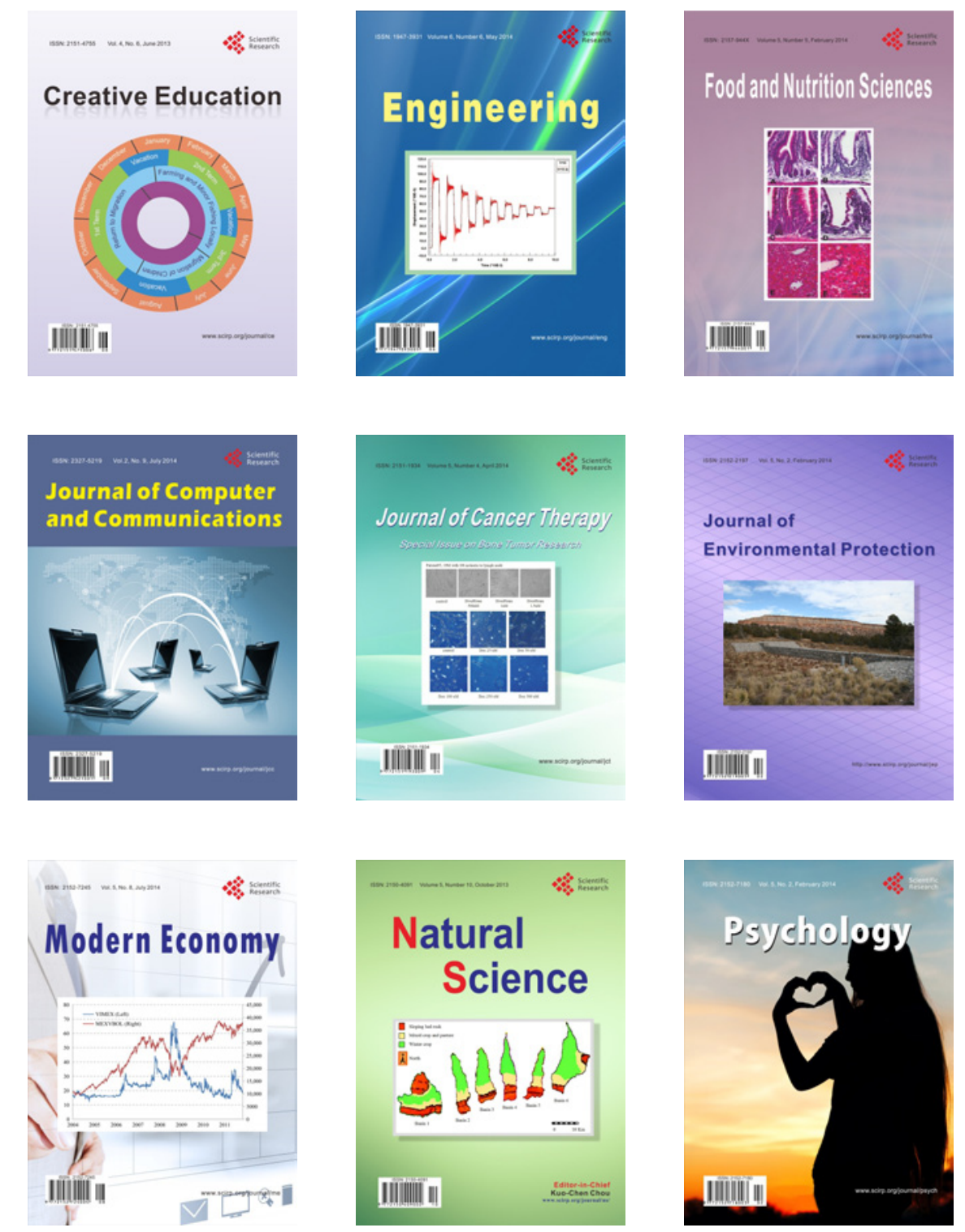